\title{
Plasma Antimicrobial Peptide LL-37 Level Is Inversely Associated with HDL Cholesterol Level in Patients with Type 2 Diabetes Mellitus
}

\author{
Shu Meguro, ${ }^{1}$ Masuomi Tomita, ${ }^{2}$ Takeshi Katsuki, ${ }^{2}$ Kiyoe Kato, ${ }^{2}$ Henpiru Oh, ${ }^{3}$ \\ Akira Ainai, ${ }^{4}$ Ryo Ito, ${ }^{4}$ Toshihide Kawai, ${ }^{1}$ Hiroshi Itoh, ${ }^{1}$ and Hideki Hasegawa ${ }^{4}$ \\ ${ }^{1}$ Division of Endocrinology, Metabolism and Nephrology, Department of Internal Medicine, School of Medicine, \\ Keio University, 35 Shinanomachi, Shinjuku-ku, Tokyo 160-8582, Japan \\ ${ }^{2}$ Department of Internal Medicine, Saiseikai Central Hospital, 1-4-17 Mita, Minato-ku, Tokyo 108-0073, Japan \\ ${ }^{3}$ Minami-Aoyama Home Clinic, 7-5-2 Minami Aoyama, Minato-ku, Tokyo 107-0062, Japan \\ ${ }^{4}$ Department of Pathology, National Institute of Infectious Disease, 1-23-1 Toyama, Shinjuku-ku, Tokyo 162-0052, Japan
}

Correspondence should be addressed to Shu Meguro; shumeg@z8.keio.jp

Received 7 January 2014; Revised 17 February 2014; Accepted 17 February 2014; Published 26 March 2014

Academic Editor: Faustino R. Pérez-López

Copyright (C) 2014 Shu Meguro et al. This is an open access article distributed under the Creative Commons Attribution License, which permits unrestricted use, distribution, and reproduction in any medium, provided the original work is properly cited.

\begin{abstract}
Introduction. Relation between atherosclerosis and innate immunity has attracted attention. As the antimicrobial peptide, LL-37, could have an important role in atherosclerosis, we supposed that there could be a meaningful association of plasma LL-37 level with risk factors for cardiovascular disease in subjects with type 2 diabetes mellitus. Materials and Methods. We evaluated plasma LL-37 level and other clinical markers in Japanese subjects with type 2 diabetes mellitus $(n=133,115$ men and 18 women; age $64.7 \pm 11.5$ years; HbAlc $8.1 \pm 1.6 \%$ ). Plasma level of LL-37 was measured by ELISA. Results. Mean plasma LL-37 level was $71.2 \pm 22.3 \mathrm{ng} / \mathrm{mL}$. Plasma LL-37 level showed significant correlations with HDL cholesterol $(r=-0.450, P<0.01)$, triglyceride $(r=0.445, P<0.01)$, and high sensitive C-reactive protein $(r=0.316, P<0.01)$ but no significant correlation with age, body mass index, HbAlc, estimated glomerular filtration rate, 25-hydroxyvitamin D, or vitamin D binding protein. Multiple linear regression analysis showed significant correlations of plasma LL-37 level with HDL cholesterol $(\beta=-0.411, P<0.01)$ and high sensitive C-reactive protein $(\beta=0.193, P<0.05)$. Conclusion. Plasma LL-37 level was positively correlated with inflammatory markers and negatively correlated with HDL cholesterol in patients with type 2 diabetes mellitus.
\end{abstract}

\section{Introduction}

Antimicrobial peptides are peptides that can kill viruses, fungi, bacteria, and other microbes [1]. They are produced in most multicellular organisms and expressed on epithelial surfaces and within circulating white cells, constitutively or in response to stimuli such as tissue injury, through interleukin-1 and other cytokines or microbial components such as lipopolysaccharide (LPS). Among such peptides, which play important roles in the innate immune system, LL37, also known as cathelicidin, is one of the most studied. In addition to its anti-infective activities, LL-37 stimulates local angiogenesis, acts synergistically with the epidermal growth factor receptor to promote epithelial growth, and attracts monocytes and neutrophils through formyl peptide receptors on these cells. In this way the peptide helps orchestrate the inflammatory process [2-4].

Innate immunity is important for primary defense not only against antimicrobial infections, but also against atherosclerosis via nonspecific inflammatory processes [5]. Involvement of LL-37 in the atherosclerotic process was suggested by previous reports. Increased transcription of LL37 was demonstrated in human atherosclerotic plaques [6], and the presence of LL-37 in human atherosclerotic lesions obtained at autopsy was shown by immunohistochemical study and it induced death of vascular smooth muscle cells [7]. A recent study in a rodent model showed that cathelicidin-related antimicrobial peptide (CRAMP: mouse 
cathelicidin equivalent to LL-37 in human) and Apo E double knockout mice exhibited reduced atherosclerotic lesions compared to Apo E knockout mice [8].

Type 2 diabetes mellitus is a worldwide epidemic and increases macrovascular and microvascular risk $[9,10]$. Residual risk for cardiovascular disease still exists in spite of widely adopted statin treatment. As one of the explanations for the residual risk of atherosclerosis, chronic subclinical inflammation is a candidate [11]. We therefore suppose that analysis of plasma LL-37 level, which is an important player in the innate immune system, in subjects with type 2 diabetes mellitus may be meaningful in regard to its association with risk factors for cardiovascular disease.

\section{Materials and Methods}

The present study was conducted according to the principles expressed in the Declaration of Helsinki. Written informed consent was obtained from each subject after a full explanation of the purpose, nature, and risk of all procedures used. The protocol was approved by the ethical review committees of Saiseikai Central Hospital, Tokyo, Japan.

We originally aimed this study to observe the association between pandemic influenza virus A/H1N1pdm09 infection and plasma 25-hydroxyvitamin D [25(OH)D] and/or plasma LL-37 level in subjects with type 2 diabetes mellitus who were considered as high risk for influenza infection. Subjects were recruited in routine outpatient clinics for their diabetes managements. Patients with hematological disorders, liver cirrhosis, malignant disease, or active gastrointestinal disease were not enrolled in this study. Patients with active inflammatory or infectious disease were excluded by their comorbidities, clinical symptoms, and body temperatures. As plasma $25(\mathrm{OH}) \mathrm{D}$ level is influenced by their renal function, we actively enrolled subjects with nephropathy to obtain equivalent numbers of subjects with each clinical stage of diabetic nephropathy according to their laboratory data at the consent to evaluate the effect of advanced stages of diabetic nephropathy. As a result, we measured plasma LL$37,25(\mathrm{OH}) \mathrm{D}$, vitamin $\mathrm{D}$ binding protein (VDBP), and other clinical markers in Japanese subjects with type 2 diabetes mellitus $(n=135,117$ men and 18 women; age $64.7 \pm 11.5$ years; HbAlc $8.1 \pm 1.6 \%$ ) who attended the outpatient clinic of Saiseikai Central Hospital between September 2009 and February 2010.

After obtaining written informed consent, blood samples in an overnight fasting state were drawn at the next visit to the usual outpatient clinic. Routine blood analysis was performed by the hospital laboratory immediately after blood sampling. After centrifugation, a part of the plasma was preserved at $-80^{\circ} \mathrm{C}$ until further analysis. Assays of fasting plasma glucose (FPG), total cholesterol, HDL cholesterol, triglyceride, serum creatinine level, and several other biochemical parameters were performed with autoanalyzers. HbAlc level was determined by high-performance liquid chromatography (Arkray Inc., Kyoto, Japan) according to the recommended method by the Japan Diabetes Society at that time and converted to the National Glycohemoglobin Standardization
Program (NGSP) value [12]. Microalbuminuria was defined as an albumin creatinine ratio (ACR) of 30 to $300 \mathrm{mg} / \mathrm{g}$ $\mathrm{Cr}$ and macroalbuminuria as ACR of more than $300 \mathrm{mg} / \mathrm{g}$ $\mathrm{Cr}$ in spot urinalysis. Estimated glomerular filtration rate (eGFR) $\left(\mathrm{mL} / \mathrm{min} / 1.73 \mathrm{~m}^{2}\right)$ was calculated using the equation provided by the Japanese Society of Nephrology as $194 \times$ $\mathrm{Cr}^{-1.094} \times$ Age $^{-0.287}$ (with further multiplication by 0.739 for female subjects) [13]. Plasma levels of LL-37, 25(OH)D, and VDBP were measured by ELISA using a human LL-37 ELISA kit (Hycult Biotechnology, Uden, Netherlands), 25(OH)vitamin D direct ELISA kit (Immundiagnostik AG, Bensheim, Germany), and vitamin D binding protein ELISA kit (Immundiagnostik AG, Bensheim, Germany), respectively, according to the manufacturers' manuals, at the laboratory of the National Institute of Infectious Diseases.

Hypertension was defined as systolic blood pressure $>140 \mathrm{mmHg}$, diastolic blood pressure $>90 \mathrm{mmHg}$, or the prescription of antihypertensive medication. Dyslipidemia was defined as LDL cholesterol $>3.63 \mathrm{mmol} / \mathrm{L}$, triglyceride $>1.72 \mathrm{mmol} / \mathrm{L}$, HDL cholesterol $<1.04 \mathrm{mmol} / \mathrm{L}$, or the prescription of lipid-lowering medication. All subjects underwent funduscopic examination by trained ophthalmologists. Clinical stage of diabetic retinopathy was classified based on the Davis classification as none, background retinopathy, and more advanced stage or previous history of photocoagulation [14]. Clinical stage of diabetic nephropathy was classified as none, microalbuminuria, macroalbuminuria, chronic renal failure (CRF), which was defined as eGFR less than $30 \mathrm{~mL} / \mathrm{min} / 1.73 \mathrm{~m}^{2}$, and dialysis. Past history of cardiovascular disease was determined by checking the medical records or detailed medical interview. Prior history of myocardial infarction, coronary intervention, ischemic stroke, or peripheral artery disease was regarded as a history of CVD.

Continuous variables are expressed as mean \pm SD. Correlation coefficients were analyzed by Pearson's test. However, triglyceride and high sensitive C-reactive protein (hs-CRP) were analyzed by Spearman's rank test because of their skewed distributions. Differences in continuous variables between the two groups were tested by Student's $t$-test, and differences among three or more groups were tested by analysis of variance (ANOVA). Multiple linear regression analysis with compulsory input was performed to evaluate the independent contribution to plasma LL-37 level. Age, sex, $\mathrm{BMI}$, disease duration, hypertension, HbA1c, HDL cholesterol, and hs-CRP were adopted as covariates. Values of $P<$ 0.05 were considered statistically significant. All analyses were performed using SPSS 21.0 statistical software (SPSS Inc., Chicago, Il, USA).

\section{Results}

Details of the patients' characteristics are shown in Table 1. Among them, $65.4 \%$ were prescribed renin-angiotensin system (RAS) blockade medication, $57.9 \%$ were prescribed a statin, and $55.6 \%$ were treated with insulin. No one revealed the signs of influenza virus infection during that season. 
TABLE 1: Clinical characteristics of study subjects.

\begin{tabular}{|c|c|}
\hline Age (years) & $64.7 \pm 11.5$ \\
\hline Sex (male/female) & $115 / 18$ \\
\hline BMI $\left(\mathrm{kg} / \mathrm{m}^{2}\right)$ & $25.6 \pm 4.5$ \\
\hline Disease duration (years) & $18.3 \pm 10.5$ \\
\hline Hypertension (\%) & 85.0 \\
\hline Dyslipidemia (\%) & 76.7 \\
\hline Smoking (\%) & 27.1 \\
\hline $\mathrm{FPG}(\mathrm{mmol} / \mathrm{L})$ & $9.6 \pm 3.1$ \\
\hline HbAlc $(\%)$ & $8.1 \pm 1.6$ \\
\hline Total cholesterol (mmol/L) & $4.8 \pm 0.8$ \\
\hline HDL cholesterol (mmol/L) & $1.3 \pm 0.4$ \\
\hline Triglyceride (mmol/L) & $1.7 \pm 1.0$ \\
\hline Plasma creatinine $(\mathrm{mmol} / \mathrm{L})$ & $183 \pm 206$ \\
\hline $\mathrm{eGFR}\left(\mathrm{mL} / \mathrm{min} / 1.73 \mathrm{~m}^{2}\right)$ & $50.4 \pm 29.1$ \\
\hline $25(\mathrm{OH}) \mathrm{D}(\mathrm{ng} / \mathrm{mL})$ & $71.2 \pm 22.3$ \\
\hline $\operatorname{VDBP}(\mathrm{mg} / \mathrm{dL})$ & $31.7 \pm 39.1$ \\
\hline hs-CRP (mg/dL) & $0.19 \pm 0.61$ \\
\hline $\mathrm{WBC}\left(\mathrm{mm}^{3}\right)$ & $7091 \pm 2058$ \\
\hline Retinopathy (none/simple/proliferative) & $51 / 53 / 29$ \\
\hline Nephropathy (none/microalbuminuria/macroalbuminuria/CRF/dialysis) & $23 / 40 / 31 / 31 / 8$ \\
\hline History of cardiovascular disease (\%) & 32.3 \\
\hline
\end{tabular}

Continuous variables are expressed as mean \pm SD. FPG: fasting plasma glucose, eGFR: estimated glomerular filtration rate, 25(OH)D: 25-hydroxyvitamin D, VDBP: vitamin D binding protein, hs-CRP: high sensitive C-reactive protein, WBC: white blood cell count, and CRF: chronic renal failure.

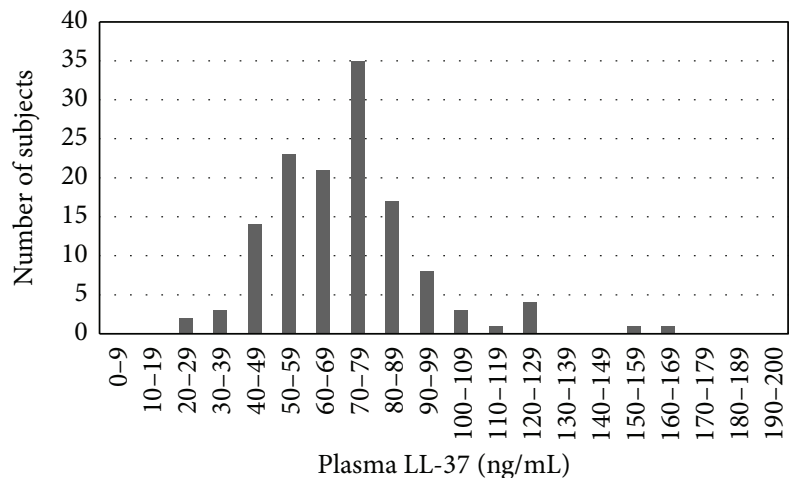

FIGURE 1: Histogram of frequency distribution of plasma LL-37 level.

Plasma LL-37 level was $71.2 \pm 22.3 \mathrm{ng} / \mathrm{mL}$ (mean \pm SD) and showed an almost normal distribution (Figure 1). Plasma LL-37 level showed significant correlations with HDL cholesterol $(r=-0.450, P<0.01)$, triglyceride $(r=0.445$, $P<0.01)$, and hs-CRP $(r=0.316, P<0.01)$, but no significant correlation with age, disease duration, BMI, HbAlc, eGFR, 25(OH)D, or VDBP (Table 2, Figure 2).

Plasma LL-37 level was not different between genders. The presence of hypertension and smoking status showed no correlation with plasma LL-37 level, but the presence of dyslipidemia showed a significant correlation with plasma LL-37 level $(P<0.01)$. The clinical stage of diabetic retinopathy did not show a significant correlation with plasma LL-37 level. The clinical stage of diabetic nephropathy did not show
TABLE 2: Correlation of plasma LL-37 level with clinical covariates.

\begin{tabular}{lcc}
\hline & $r$ & $P$ value \\
\hline Age & -0.161 & n.s. \\
BMI & 0.128 & n.s. \\
Disease duration & -0.165 & n.s. \\
Plasma creatinine & 0.070 & n.s. \\
eGFR & -0.098 & n.s. \\
Uric acid & 0.082 & n.s. \\
Total cholesterol & 0.073 & n.s. \\
HDL cholesterol & -0.450 & $<0.01$ \\
LDL cholesterol & 0.132 & n.s. \\
Triglyceride & 0.445 & $<0.01$ \\
FPG & 0.027 & n.s. \\
HbAlc & 0.085 & n.s. \\
hs-CRP & 0.316 & $<0.01$ \\
WBC & 0.162 & n.s. \\
25(OH)D & -0.164 & n.s. \\
VDBP & 0.092 & n.s. \\
\hline
\end{tabular}

eGFR: estimated glomerular filtration rate, FPG: fasting plasma glucose, hs-CRP: high sensitive $\mathrm{C}$ reactive protein, WBC: white blood cell count, 25(OH)D: 25-hydroxyvitamin D, and VDBP: vitamin D binding protein. n.s.: not significant.

a tendency for a relation with LL-37 level. Plasma LL-37 level did not differ according to history of cardiovascular disease. Treatment with a RAS blocker, statin, or insulin did not show a significant difference between the groups either. HDL cholesterol level did not differ according to history of 


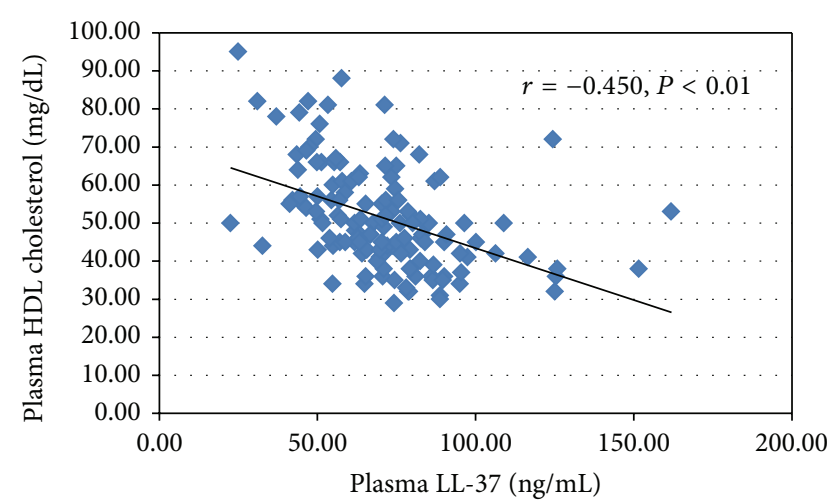

(a) HDL cholesterol and plasma LL-37 $r=-0.450, P<0.01$, by Pearson's test

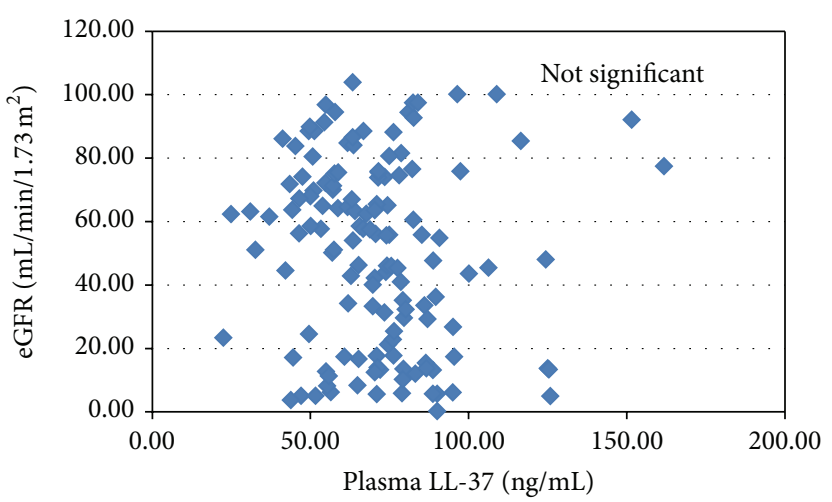

(c) eGFR and plasma LL-37, not significant, by Pearson's test

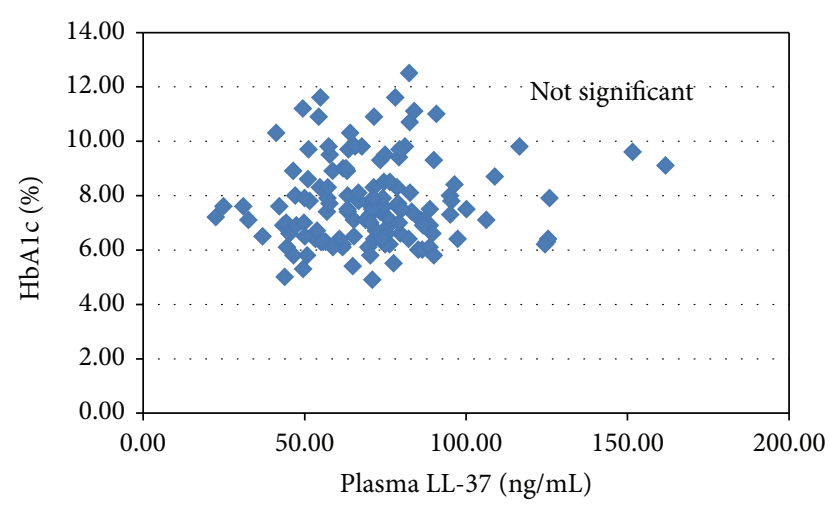

(b) HbAlc and plasma LL-37, not significant, by Pearson's test

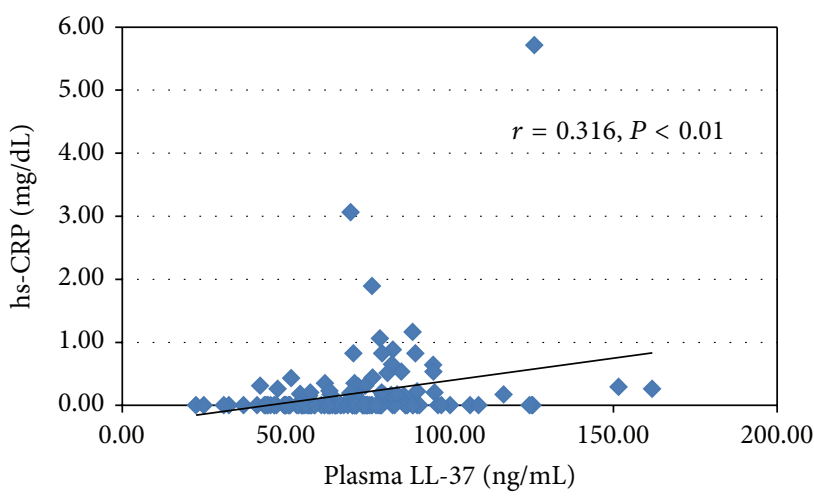

(d) hs-CRP and plasma LL-37 $r=0.316, P<0.01$, by Spearman's test

Figure 2: Plasma LL-37 level and covariates.

cardiovascular disease, and statins were frequently prescribed in subjects with a history of cardiovascular disease (chi squared analysis, $P<0.01$ ).

Multiple linear regression analysis showed significant correlations of plasma LL-37 level with HDL cholesterol $(\beta=$ $-0.411, P<0.01)$ and hsCRP $(\beta=0.193, P<0.05)$ (Table 3$)$.

\section{Discussion}

This study demonstrated that plasma LL-37 level was positively correlated with inflammatory markers and negatively correlated with HDL cholesterol in patients with type 2 diabetes mellitus. These results are very interesting in view of several recent reports that elucidated an association between innate immunity and atherosclerosis.

There was a gender disproportion in this study subjects although there was not a statistical difference in plasma LL37 level between genders. This occurred unintentionally in our recruiting process. It was partly because of the patient population of our hospital (more than two-third were male). Also, although we aimed to obtain equivalent numbers of subjects with each clinical stage of diabetic nephropathy, the number of the subjects without microalbuminuria was smaller than the other stages of nephropathy. This occurred by the visit to visit variation of ACR values. As the plasma level of LL-37 was not different between genders and among
TABLE 3: Multiple linear regression analysis of plasma LL-37 level.

\begin{tabular}{lcc}
\hline$R^{2}$ & & 0.283 \\
Variables & Beta & $P$ value \\
\hline Age & -0.156 & n.s. \\
Sex & -0.131 & n.s. \\
BMI & -0.116 & n.s. \\
Disease duration & -0.124 & n.s. \\
Hypertension & -0.120 & n.s. \\
HbAlc & 0.024 & n.s. \\
HDL cholesterol & -0.411 & $<0.01$ \\
hs-CRP & 0.193 & $<0.05$ \\
\hline
\end{tabular}

Beta is the standardized partial regression coefficient of multiple linear regression analysis. n.s.: not significant.

the clinical stages of nephropathy, we did not consider that this disproportion of the study population affected the results.

Type 2 diabetes mellitus is a major risk factor for cardiovascular disease $[15,16]$. Reduced HDL cholesterol and increased triglyceride are typical features of dyslipidemia in type 2 diabetes mellitus [17]. Although recent large interventional clinical trials in type 2 diabetes mellitus failed to show a reduction in mortality or cardiovascular events [1820], residual risk for cardiovascular disease still exists in spite of widely adopted statin treatment. Interventions on 
HDL cholesterol are one of the most promising treatments for residual risk for cardiovascular disease [21, 22]; however, some studies of cholesterol ester transfer protein (CETP) inhibitors, which increase plasma HDL cholesterol level, failed to show a protective effect against cardiovascular disease [23-25]. As a result, the mechanism of the preventive effect of HDL cholesterol on the atherosclerotic process is still being researched [26], even though it is now accepted that a higher HDL cholesterol level is beneficial in terms of cardiovascular disease. In this context, the relation between HDL cholesterol and immunity is receiving a lot of attention [27]. For example, apoprotein A-I, which is one main apoprotein in HDL, has a protective effect from lipopolysaccharide (LPS) toxicity [27]. The lipid composition change of lipid rafts on cell membrane occurs by the cholesterol efflux through the interaction between HDL and lipid rafts. This change seems to modulate the series of immune responses, including monocytes, macrophages, and B and T lymphocytes [28]. HDL also transports several immunologically active substances, such as sphingosine-1-phosphate $[27,28]$. We did not evaluate apoprotein fractions in this study so we could not assess if apoprotein composition in HDL affect our results. However, we consider that our results add new evidence for a link between HDL cholesterol and immunity.

Chronic inflammation is involved in the pathogenesis of CVD. Several prospective studies indicated that an elevated hs-CRP level is a risk factor for CVD [29-32]. Randomized control study by the usage of statin also revealed that the achievements of lower levels of both the LDL cholesterol and the hs-CRP are independently related to the incident of CVD $[33,34]$. The correlation between hs-CRP and LL-37, which we demonstrated in this study, might suggest the association of innate immune process with the chronic inflammation and elevated hs-CRP level.

As written in Section 1, LL-37 has multifactorial functions such as antimicrobial effect, antiviral effect, as well as induction of chemotaxis and stimulation of cytokine release [1-4]. LL-37 indirectly attracts more immune cells by inducing secretion of IL- 8 from macrophages, fibroblasts, and epithelial cells [3]. LL-37 also induces expression of the adhesion molecule intercellular adhesion molecule-1 and the chemokine monocyte chemoattractant protein 1 in endothelial cells and induces the death of smooth muscle cells [6]. This series of phenomenon induced by LL-37 has been considered important in progression and rupture of atheromatous plaque. LL-37 might play a pivotal role in this series of process interactively with HDL. We suppose that the role of LL-37 in atherosclerosis should be studied further.

In this study, plasma LL-37 level was associated with HDL cholesterol level but not with a history of cardiovascular disease or statin treatment. However, statins are frequently prescribed in patients with a history of cardiovascular disease. As plasma HDL cholesterol level was not different regardless of history of cardiovascular disease, subjects with a history might have shown lower HDL cholesterol levels without statin treatment.

It is also known that LL-37 is induced by vitamin D, and the gene encoding LL-37 contains sites for the vitamin D receptor [1]. So the link between vitamin D and innate immunity is also being researched $[35,36]$. In our study, plasma $25(\mathrm{OH}) \mathrm{D}$ level and VDBP level were not associated with plasma LL-37 level. One of the characteristics of our study subjects is that we included many subjects with advanced stages of diabetic nephropathy, which made mean 25(OH)D level in our study relatively low. There must be a complicated mechanism for the association between vitamin D and LL-37 involving obesity, diabetes, dyslipidemia, renal impairment, and the immune system. We cannot explain the reason for this dissociation, but the characteristics of the study group might have influenced the results.

There are some limitations of this study. One is that it had a cross-sectional design and was performed at one facility, so the results should be confirmed at other facilities in the future. Most subjects in this study had long duration of diabetes and relatively advanced complications. As duration of diabetes did not influence the level of plasma LL-37, we suppose we can expect the same relation in the subjects with shorter duration of disease, but it should be confirmed in the future. As another limitation, the clinical significance of plasma LL-37 level is still not certain. A complicated mechanism might exist in the association between HDL cholesterol and plasma LL-37 level. However, this study showed a correlation between plasma HDL cholesterol level and plasma LL-37 level. Further research is needed to clarify the relation between atherosclerosis and immunity, in order to reduce the residual risk for cardiovascular disease.

\section{Conflict of Interests}

This study was supported by the grant form Ministry of Health, Welfare, and Labor in Japan. The funders had no role in study design, data collection and analysis, decision to publish, or preparation of the paper.

\section{References}

[1] M. Zasloff, "Fighting infections with vitamin D," Nature Medicine, vol. 12, no. 4, pp. 388-390, 2006.

[2] M. Zasloff, "Antimicrobial peptides of multicellular organisms," Nature, vol. 415, no. 6870, pp. 389-395, 2002.

[3] D. Vandamme, B. Landuyt, W. Luyten, and L. Schoofs, "A comprehensive summary of LL-37, the factotum human cathelicidin peptide," Cellular Immunology, vol. 280, no. 1, pp. 22-35, 2012.

[4] R. Bucki, K. Leszczyńska, A. Namiot, and W. Sokołowski, "Cathelicidin LL-37: a multitask antimicrobial peptide," Archivum Immunologiae et Therapiae Experimentalis, vol. 58, no. 1, pp. 15-25, 2010.

[5] A. H. Lichtman, C. J. Binder, S. Tsimikas, and J. L. Witztum, "Adaptive immunity in atherogenesis: new insights and therapeutic approaches," Journal of Clinical Investigation, vol. 123, no. 1, pp. 27-36, 2013.

[6] K. Edfeldt, B. Agerberth, M. E. Rottenberg et al., "Involvement of the antimicrobial peptide LL-37 in human atherosclerosis," Arteriosclerosis, Thrombosis, and Vascular Biology, vol. 26, no. 7, pp. 1551-1557, 2006.

[7] C. D. Ciornei, H. Tapper, A. Bjartell, N. H. Sternby, and M. Bodelsson, "Human antimicrobial peptide LL-37 is present in 
atherosclerotic plaques and induces death of vascular smooth muscle cells: a laboratory study," BMC Cardiovascular Disorders, vol. 6, article 49, 2006.

[8] Y. Döring, M. Drechsler, S. Wantha et al., "Lack of neutrophilderived CRAMP reduces atherosclerosis in mice," Circulation Research, vol. 110, no. 8, pp. 1052-1056, 2012.

[9] C. J. Nolan, P. Damm, and M. Prentki, “Type 2 diabetes across generations: from pathophysiology to prevention and management," The Lancet, vol. 378, no. 9786, pp. 169-181, 2011.

[10] M. Stolar, "Glycemic control and complications in type 2 diabetes mellitus," The American Journal of Medicine, vol. 123, no. 3, pp. S3-S11, 2010.

[11] T. Mazzone, A. Chait, and J. Plutzky, "Cardiovascular disease risk in type 2 diabetes mellitus: insights from mechanistic studies," The Lancet, vol. 371, no. 9626, pp. 1800-1809, 2008.

[12] A. Kashiwagi, M. Kasuga, E. Araki et al., "International clinical harmonization of glycated hemoglobin in Japan: from Japan diabetes society to national glycohemoglobin standardization program values," Journal of Diabetes Investigation, vol. 3, no. 1, pp. 39-40, 2012.

[13] S. Matsuo, E. Imai, M. Horio et al., "Revised equations for estimated GFR from serum creatinine in Japan," The American Journal of Kidney Diseases, vol. 53, no. 6, pp. 982-992, 2009.

[14] D. Davis, T. Kern, and L. I. Rand, International Textbook of Diabetes Mellitus, Diabetic Retinopathy, John Wiley \& Sons, Chichester, UK, 2nd edition, 1997.

[15] S. M. Haffner, S. Lehto, T. Rönnemaa, K. Pyörälä, and M. Laakso, "Mortality from coronary heart disease in subjects with type 2 diabetes and in nondiabetic subjects with and without prior myocardial infarction," The New England Journal of Medicine, vol. 339, no. 4, pp. 229-234, 1998.

[16] T. Nishimura, K. Nakajima, H. Kusuoka, A. Yamashina, and S. Nishimura, "Prognostic study of risk stratification among Japanese patients with ischemic heart disease using gated myocardial perfusion SPECT: J-ACCESS study," European Journal of Nuclear Medicine and Molecular Imaging, vol. 35, no. 2, pp. 319-328, 2008.

[17] S. Lehto, T. Rönnemaa, S. M. Haffner, K. Pyörälä, V. Kallio, and M. Laakso, "Dyslipidemia and hyperglycemia predict coronary heart disease events in middle-aged patients with NIDDM," Diabetes, vol. 46, no. 8, pp. 1354-1359, 1997.

[18] H. C. Gerstein, M. E. Miller, R. P. Byington et al., "Effects of intensive glucose lowering in type 2 diabetes," The New England Journal of Medicine, vol. 358, no. 24, pp. 2545-2559, 2008.

[19] A. Patel, S. MacMahon, J. Chalmers et al., "Intensive blood glucose control and vascular outcomes in patients with type 2 diabetes," The New England Journal of Medicine, vol. 358, no. 24, pp. 2560-2572, 2008.

[20] W. Duckworth, C. Abraira, T. Moritz et al., "Glucose control and vascular complications in veterans with type 2 diabetes," The New England Journal of Medicine, vol. 360, no. 2, pp. 129-139, 2009.

[21] C. Cimminiello, "What intervention trials don't tell us: the residual risk in primary prevention," Internal and Emergency Medicine, vol. 6, no. 1, supplement, pp. 53-60, 2011.

[22] A. Zambon, "Residual cardiovascular risk in secondary prevention," Internal and Emergency Medicine, vol. 6, no. 1, supplement, pp. 61-68, 2011.

[23] G. G. Schwartz, A. G. Olsson, M. Abt et al., "Effects of dalcetrapib in patients with a recent acute coronary syndrome," The New England Journal of Medicine, vol. 367, no. 22, pp. 20892099, 2012.
[24] S. E. Nissen, J. Tardif, S. J. Nicholls et al., "Effect of torcetrapib on the progression of coronary atherosclerosis," The New England Journal of Medicine, vol. 356, no. 13, pp. 1304-1316, 2007.

[25] P. J. Barter, M. Caulfield, M. Eriksson et al., "Effects of torcetrapib in patients at high risk for coronary events," The New England Journal of Medicine, vol. 357, no. 21, pp. 2109-2122, 2007.

[26] H. B. Brewer Jr., "Clinical review: the evolving role of HDL in the treatment of high-risk patients with cardiovascular disease," Journal of Clinical Endocrinology and Metabolism, vol. 96, no. 5, pp. 1246-1257, 2011.

[27] G. D. Norata, A. Pirillo, E. Ammirati, and A. L. Catapano, "Emerging role of high density lipoproteins as a player in the immune system," Atherosclerosis, vol. 220, no. 1, pp. 11-21, 2012.

[28] M. G. Sorci-Thomas and M. J. Thomas, "High density lipoprotein biogenesis, cholesterol efflux, and immune cell function," Arteriosclerosis, Thrombosis, and Vascular Biology, vol. 32, no. 11, pp. 2561-2565, 2012.

[29] P. M. Ridker, C. H. Hennekens, J. E. Buring, and N. Rifai, "C-reactive protein and other markers of inflammation in the prediction of cardiovascular disease in women," The New England Journal of Medicine, vol. 342, no. 12, pp. 836-843, 2000.

[30] J. Danesh, J. G. Wheeler, G. M. Hirschfield et al., "C-reactive protein and other circulating markers of inflammation in the prediction of coronary heart disease," The New England Journal of Medicine, vol. 350, no. 14, pp. 1387-1397, 2004.

[31] Emerging Risk Factors Collaboration, S. Kaptoge, E. di Angelantonio et al., "C-reactive protein concentration and risk of coronary heart disease, stroke, and mortality: an individual participant meta-analysis," The Lancet, vol. 375, no. 9709, pp. 132-140, 2010.

[32] H. Arima, M. Kubo, K. Yonemoto et al., "High-sensitivity c-reactive protein and coronary heart disease in a general population of Japanese: the Hisayama study," Arteriosclerosis, Thrombosis, and Vascular Biology, vol. 28, no. 7, pp. 1385-1391, 2008.

[33] P. M. Ridker, E. Danielson, F. A. H. Fonseca et al., "Rosuvastatin to prevent vascular events in men and women with elevated Creactive protein," The New England Journal of Medicine, vol. 359, no. 21, pp. 2195-2207, 2008.

[34] P. M. Ridker, E. Danielson, F. A. Fonseca et al., "Reduction in Creactive protein and LDL cholesterol and cardiovascular event rates after initiation of rosuvastatin: a prospective study of the JUPITER trial," The Lancet, vol. 373, no. 9670, pp. 1175-1182, 2009.

[35] Q. Wang, W. Zhang, H. Li et al., "Effects of 25-hydroxyvitamin D3 on cathelicidin production and antibacterial function of human oral keratinocytes," Cell Immunology, vol. 283, no. 1-2, pp. 45-50, 2013.

[36] H. B. Nguyen, B. Eshete, K. H. Lau, A. Sai, M. Villarin, and D. Baylink, "Serum 1, 25-dihydroxyvitamin D: an outcome prognosticator in human sepsis," PLoS ONE, vol. 8, no. 5, Article ID e64348, 2013. 


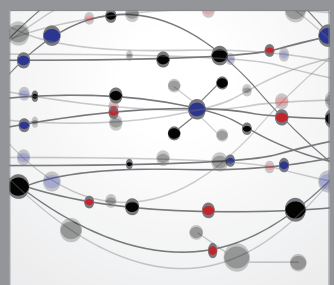

The Scientific World Journal
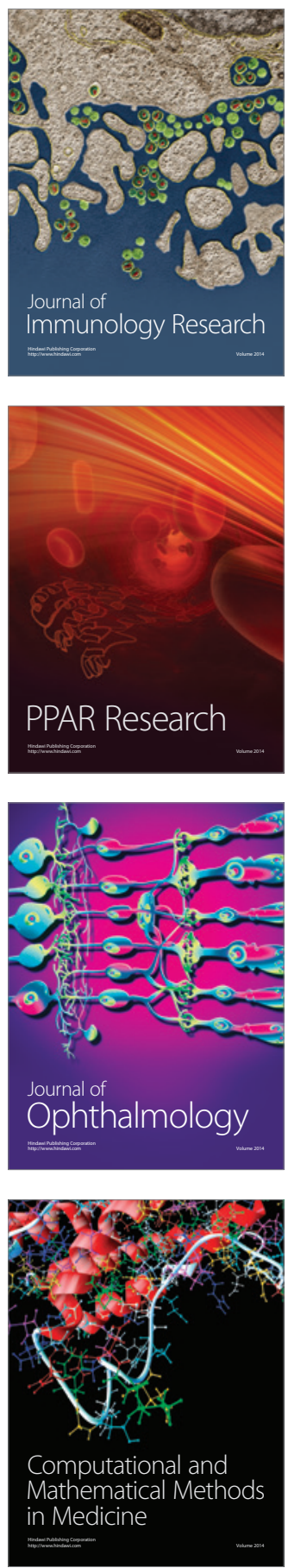

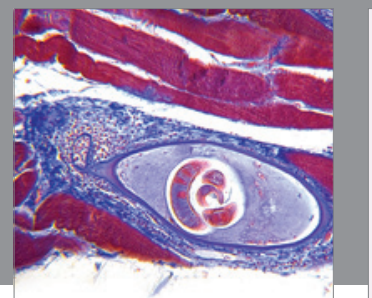

Gastroenterology

Research and Practice
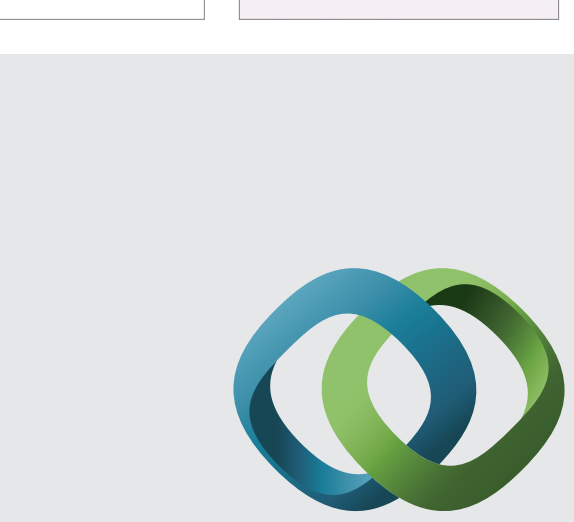

\section{Hindawi}

Submit your manuscripts at

http://www.hindawi.com
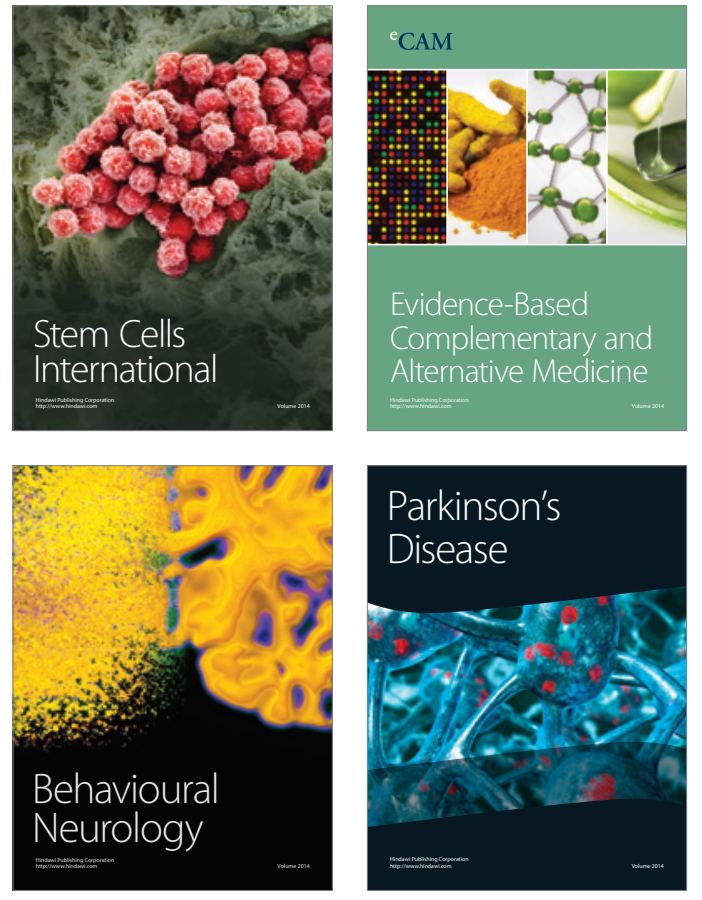
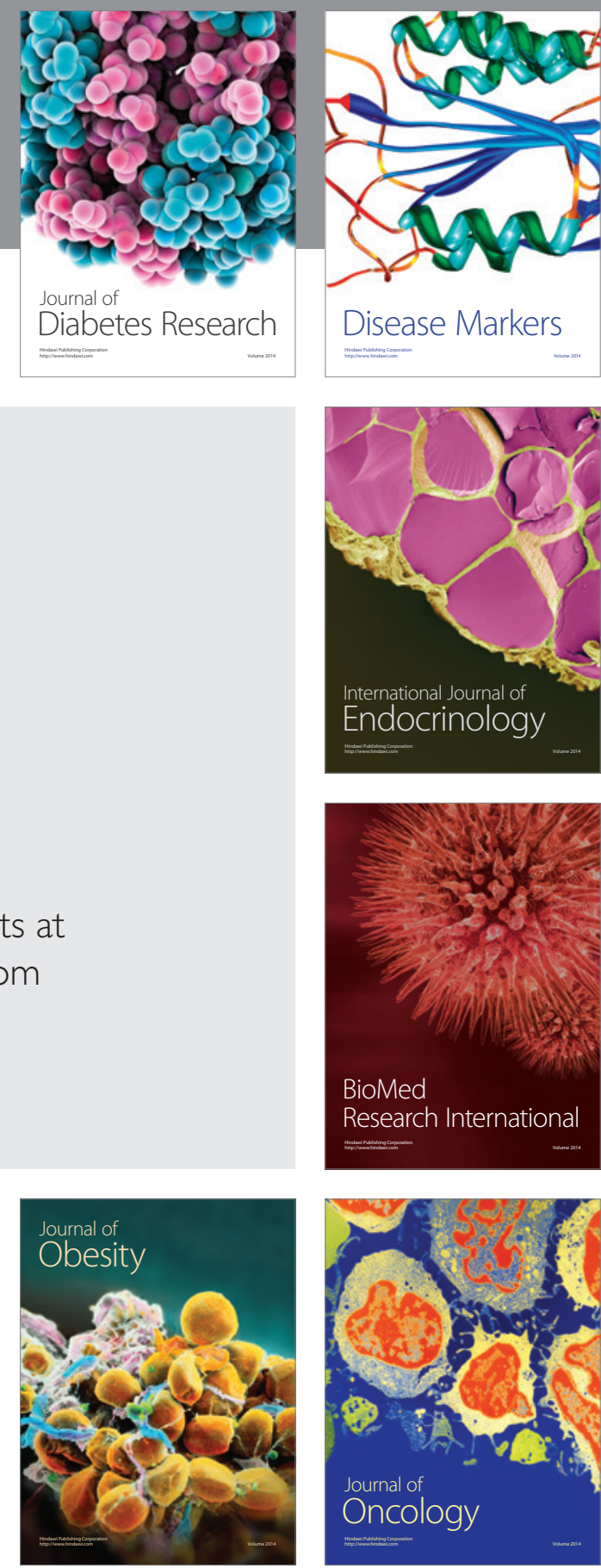

Disease Markers
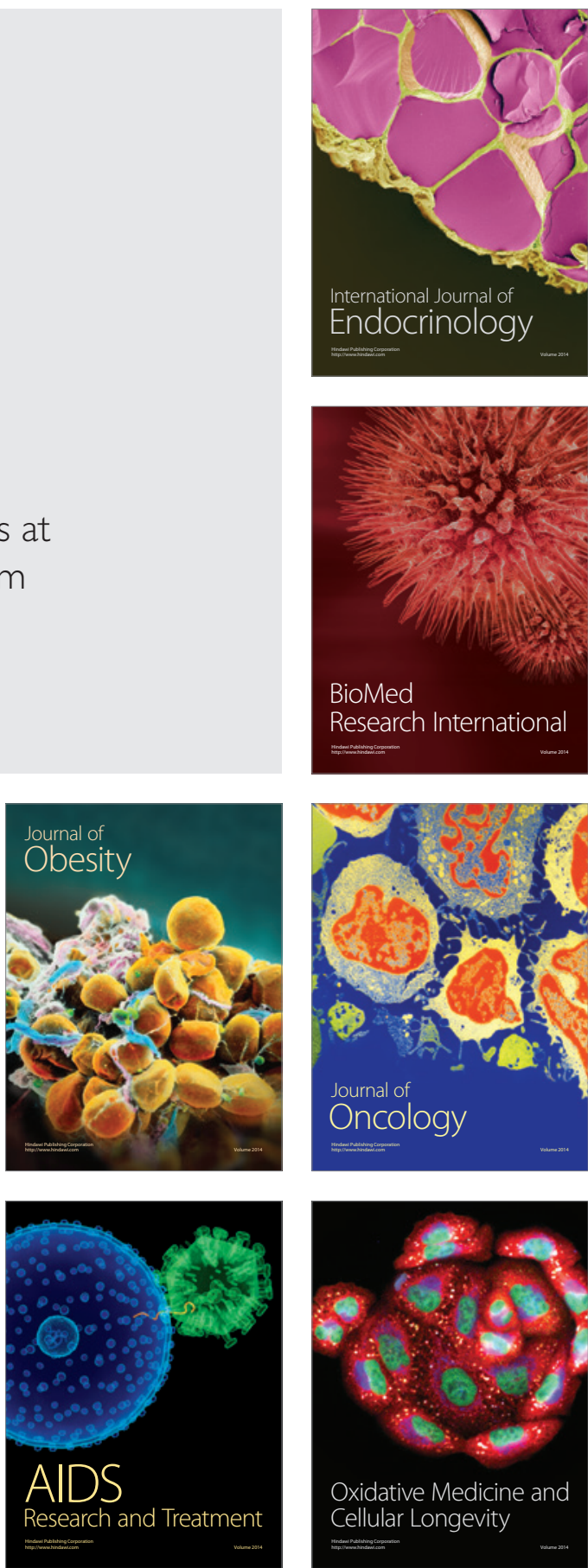